\title{
Fault Analysis Research on Electrical System of Equipment for Tracking and Controlling
}

\author{
Zheng Liu, Bo Zhang \\ China Satellite Tracking and Controlling Department, Jiangyin 214431, China \\ liuzheng354@qq.com
}

\begin{abstract}
Keywords: equipment for Tracking and controlling; electrical system; fault analysis
Abstract. The electrical system is an important component of equipment for tracking and controlling, so its fault will have a great influence on the performance of equipment for tracking and controlling. The electrical system structure of equipment for tracking and controlling is complex, with various types, so its fault detection is pretty difficult. To improve the efficiency of fault detection, multiple detection methods in professional group have improved the maintenance support capability of electrical system to some certain extent. Aimed at the problems on aspects like the existence of low automatic degree of current testing equipment in electrical system of equipment for tracking and controlling, its weak versatility and insufficient serialization specification, etc., In the Paper, the electrical system of equipment for tracking and controlling is taken as the research object to analyze and research the fault of the electrical system.
\end{abstract}

\section{Introduction}

According to different functions, the electrical system of equipment for tracking and controlling can be divided into power-supply system, servo motor system, voltage regulation system, auxiliary electrical installation and detection apparatus, characteristics of which are various kinds and amount, different structure functions and wide distribution. The improvement for digitization and informationization degree of electrical system of equipment for tracking and controlling significantly improves the performance characteristic of equipment for tracking and controlling[1]. The application of a large number of electronic devices and electrical equipment has increased the probability of fault of the electrical system, which poses a problem to improve the maintenance of the electrical system so as to make the fault detection of electrical system more efficient and the fault location more accurate, so the fault analysis method for electrical system is especially important.

\section{Structure composition and fault characteristics of electrical system of equipment for tracking and controlling}

At present, the operation of testing equipment in electrical system of equipment for tracking and controlling is rather tedious and time-consuming of detection is rather long, in which its automatic degree is rather low and it is lack of the capabilities like information records, information storage and information management, so it has higher demanding for the operator's professional and technical competence. Secondly, the testing equipment in electrical system is unreasonably equipped, so their versatility is poor and comprehensive integration degree is low. The detection degree for testing equipment of electrical system is not considered under the current maintenance support system[2].

\section{Structure composition of electrical system}

Power-supply system. the power-supply system of equipment for tracking and controlling is to guarantee that it can stably keep supplying power to electric equipment under various service conditions, which mainly ensures the stable pressure by isolation transformer and UPS system so as to guarantee the normal operation of each electric device.

Electric device. The electric device accomplishes the corresponding functions by consuming electric energy, which mainly includes servo motor system, industrial control computer and various heating and lighting system, etc. and equipment. 
Detecting instrument. The detecting instrument is mainly used to indicate and inspect signal status and working conditions of motor systems and electrical equipment, which mainly includes: photoelectric encoder, gyroscope, resolver and thermometers, etc.

\section{Fault characteristics of electrical system}

The main characteristics of electrical system fault include: level, communicability, interdependency, delay, radioactivity and uncertainty.

Level. The electric device accomplishes the corresponding functions by consuming electric energy, which mainly includes servo motor system, industrial control computer and various heating and lighting system, etc. and equipment.

Communicability. There are mainly two modes of communicability, one of which is horizontal communication and the other one of which is vertical communication. Horizontal communication is defined as fault communication mode among the same level; longitudinal communication is defined as fault communication mode of modules, components, subsystems and systems arising from fault sequence of parts.

Interdependency means that a fault cause may correspond to several fault phenomena, in which some fault phenomenon may arise from multiple fault causes.

Delay means the delay of fault occurrence and development in time.

Radioactivity means fault on some part of electrical system, but the performance is not significant in its own, while it is reflected on the other parts.

Uncertainty means the cause and phenomenon of the fault is blurred, because the randomness of fault has the characteristics of uncertainty.

\section{Fault analysis on electrical system of equipment for tracking and controlling}

In recent years, with the continuous development of artificial intelligence technology and the computer technology, they have provided a new theoretical basis for fault diagnosis technology, hence, a lot of fault diagnosis methods based on knowledge have been produced, in which the prominent characteristics of these methods are that the relevant problems of fault diagnosis can be solved without the requirements to know the specific mathematical model of research object and this kind of methods mainly includes: neural network fault diagnosis method[3], fuzzy fault diagnosis method, information fusion fault diagnosis method[4] and fault tree fault diagnosis method[5], etc.

Neural network fault diagnosis method. Neural network is formed by extensive and complex connection of a large number of neurons, which is the simulation for structure and function of nerve cells in human brain and has the similar capabilities like learning, association and memory, etc to human brain. However, the core technology of fault diagnosis is pattern recognition of fault, because neural network itself has parallelism, self-learning, self-organization and associative memory function, etc.

Fuzzy fault diagnosis method. Fuzzy fault diagnosis method is to use subordinating degree function and fuzzy relation matrix in fuzzy set to express the relationship between the fault mode and the fault symptom together so as to realize the detection and diagnosis of fault, in which the conclusion got from this method is clear and intuitive and the calculation is simple and convenient.

Information fusion fault diagnosis method. Information fusion is to comprehensively analyze information from multiple sensors and handle data with computer in accordance with a certain criterion so as to complete the required decision and decision outcome. The main reason for the application of information fusion in fault diagnosis is as follows: firstly, different channel signals are formed due to multiple sensors; secondly, the same signal has formed information with different characteristics; thirdly, diagnosis outcome with deviation has appeared in different diagnosis approach. The ultimate goal of this approach is to use all kinds of information to improve the accuracy of diagnosis.

Fault tree fault diagnosis method. Fault tree fault diagnosis is a behavioral model based on the structure and function of the diagnosed object and a qualitative causal model. Its top event is the event 
that the system does not want to occur, other events that can result in the occurrence of top event are called intermediate events and bottom events and the inverted tree structure among events shall be expressed with logic gate. The fault tree model has embodied the logical relationship between feature vector and fault causes.

\section{Detection method research based on fault tree fault diagnosis}

Fault tree is a special inverted-tree building-block of logic, whose prominent characteristics are strong level and logical. Because it can reflect the relationship between faults, it is pretty suitable for fault analysis on electrical system of equipment for tracking and controlling. Fault tree analysis (FTA) method was first proposed by Watson and Haasl from Bell Labs, America in 1961, which was a useful tool for fault analysis on system, mainly including two aspects: qualitative analysis and quantitative analysis, where qualitative analysis means: to find the event causes and cause combination that should not occur in system, that is, to find all fault modes resulting in the occurrence of top events and quantitative analysis means: when the probability of all bottom events is given, it can calculate the probability of occurrence of top events and other quantitative indexes.

Basic principle of fault tree analysis. Related concepts are as follows:

Definition 1: event.An event means the description of the state of a system, module and component. The normal event is the state of affairs that can complete the corresponding functions, while the fault event is the state of affairs that can not complete the corresponding functions.Definition 2: fault tree component.All elements, components, equipments, subsystems, human factors and environmental factors, etc. that can produce fault events are called fault tree components.Definition 3: structure function. The structure function of fault tree is set as $\Phi(x)$, where

$$
\Phi(x)=\Phi\left(x_{1}, x_{2}, \mathrm{~L}, x_{n}\right)=\left\{\begin{array}{lc}
1 & \text { occurrence of top events } \\
0 & \text { nonoccurrence of top events }
\end{array}\right.
$$

In the formula, $x_{i}(i=1,2, \mathrm{~L} n)$ means the state of bottom event $x_{i}$.

$$
x_{i}= \begin{cases}1 & \text { occurrence of bottom events } \\ 0 & \text { nonoccurrence of top events }\end{cases}
$$

To make it convenient for expression, $\left(x_{1}, x_{2}, \mathrm{~L} x_{i-1}, 1, x_{i+1}, \mathrm{~L} x_{n}\right)$ will be recorded as $\left(1_{i}, X\right)$ and $\left(x_{1}, x_{2}, \mathrm{~L} x_{i-1}, 0, x_{i+1}, \mathrm{~L} x_{n}\right)$ will be recorded as $\left(0_{i}, X\right)$. If $\Phi\left(1_{i}, X\right)=\Phi\left(0_{i}, X\right), x_{i}$ is unrelated to structure function $\Phi(x)$, that is, whether the fault occurs or not, it does not have any influence on top events. On the contrary, $x_{i}$ is related to structure function $\Phi(x)$.

Qualitative analysis on fault tree of electrical system. The thought of fault tree of t electrical system is that: first, confirm the top events, establish boundary conditions and obtain original fault tree by step-by-step analysis and then simply the original fault tree to obtain the final fault tree to provide convenience for follow-up analysis research. The basic steps to build a tree are shown in Figure 1:

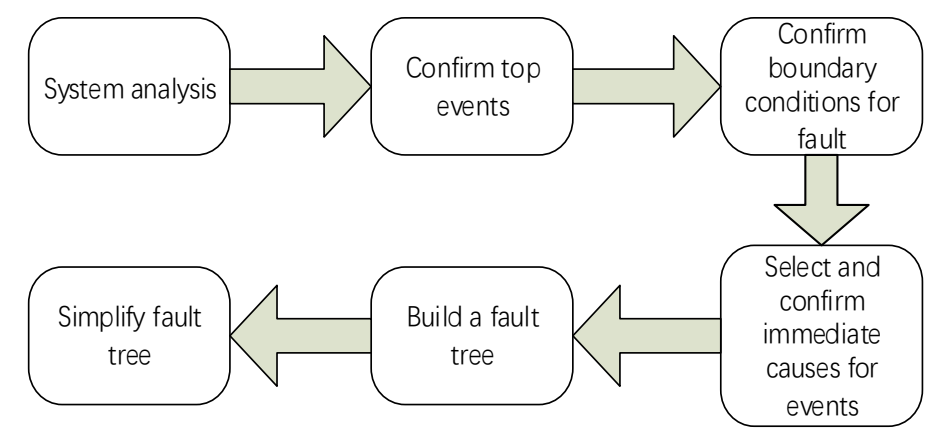

Fig. 1 Step Block Diagram for Building Fault Tree 
Confirm the top event: top event itself is a system-level failure event. On the basis of a comprehensive analysis on the system, distinction between the important and the lesser one shall be achieved and no fault events shall be missed. Conform boundary conditions: prior to establishing a fault tree, the analyzed system shall be clear in accordance with analyzed goals so that an equivalent simplified system diagram with logic relationship can be obtained from real system diagram. The fault on the last level shall be analyzed to find the direct causes in accordance with the fault on the last level and the step-by-step downward finding shall not be stopped until each bottom event are found, so a fault tree can be obtained.

\section{Conclusions}

The electrical system of equipment for tracking and controlling is a complex system with strong comprehensiveness. With the reality of increasing complexity of the electrical system of modern equipment for tracking and controlling and the significant improvement of automatic degree, the fault detection of electrical system is also more and more difficult. To make it convenient for use and improve efficiency, find the optimal detection flow for the electrical system fault with the same kinds by deeply analyzing the electrical system fault of equipment for tracking and controlling and distinguishing the fault characteristics of electrical equipment to provide theoretical support for the comprehensive integration of testing equipment in electrical system of equipment for tracking and controlling.

\section{References}

[1] Zhu Daqi. Fault Diagnosis Principle and Practice for Electronic Equipment [M]. Beijing: Publishing House of Electronics Industry, 2004.01.

[2] Huang Yanni. Development for Detection System of Some Type of UAV Ground Control Station [D]. Master Degree Thesis of Nanjing University of Aeronautics and Astronautics, 2012.

[3] Xu Liangliang. Research on Fault Diagnosis of Induction Motor Based on Neural Network[D]. Master Degree Thesis of Yangzhou University, May 2009.

[4] Shen Huairong. Information Fusion Fault Diagnosis Technology[M]. Beijing: Science Press, June 2013.

[5] Liu Dong. Dynamic Fault Tree Analysis Method [M]. Beijing: National Defense Industry Press, November 2013. 